\title{
Tuboovarian Abscess due to Colonic Diverticulitis in a Virgin Patient with Morbid Obesity: A Case Report
}

\author{
Zafer Selçuk Tuncer, ${ }^{1}$ Gokhan Boyraz, ${ }^{2}$ Senem Özge Yücel, ${ }^{2}$ \\ İlker Selçuk, ${ }^{2}$ and Aslıhan Yazicioğlu' ${ }^{2}$ \\ ${ }^{1}$ Gynecologic Oncology Unit, Department of Obstetrics and Gynecology, Hacettepe University Faculty of Medicine, \\ 06100 Ankara, Turkey \\ ${ }^{2}$ Department of Obstetrics and Gynecology, Hacettepe University Faculty of Medicine, 06100 Ankara, Turkey
}

Correspondence should be addressed to Gokhan Boyraz,gokhan.boyraz@gmail.com

Received 17 June 2012; Accepted 22 July 2012

Academic Editor: Faustino R. Pérez-López

Copyright ( 2012 Zafer Selçuk Tuncer et al. This is an open access article distributed under the Creative Commons Attribution License, which permits unrestricted use, distribution, and reproduction in any medium, provided the original work is properly cited.

\begin{abstract}
Since tuboovarian abscess is almost always a complication of pelvic inflammatory disease, it is rarely observed in virgins. A 30 year-old virgin patient presented with pelvic pain, fever, and vaginal spotting for the previous three weeks. Her abdominopelvic computed tomography scan revealed bilateral multiseptated cystic masses with prominent air-fluid levels suggesting tuboovarian abscesses. The sigmoid colon was lying between two tuboovarian masses, and its borders could not be distinguished from the ovaries. The patient was presumed to have bilateral tuboovarian abscesses which developed as a complication of the sigmoid diverticulitis. She was administered intravenous antibiotic therapy followed by percutaneous drainage under ultrasonographic guidance. She was discharged on the twenty second day with prominent clinical and radiological improvement. Diverticulitis may be a reason for development of tuboovarian abscess in a virgin patient. Early recognition of the condition with percutaneous drainage in addition to antibiotic therapy helps to have an uncomplicated recovery.
\end{abstract}

\section{Introduction}

A tuboovarian abscess reflects an agglutination of pelvic organs including the tube, ovary, and bowel forming a palpable complex. It represents an end-stage process of acute pelvic inflammatory disease. Tuboovarian abscess has been reported to complicate $18-34 \%$ of patients with pelvic inflammatory disease $[1,2]$. Risk factors for tuboovarian abscess are similar to that of pelvic inflammatory disease and include multiple sexual partners, intrauterine device, and low socioeconomic status [3]. Since the disease commonly is caused by the sexually transmitted microorganisms, intercourse with a partner having infection is the most important risk factor in tuboovarian abscess formation. However, gynecologic surgery, genital malignancy, in vitro fertilization treatment, and perforated appendicitis have also been shown to cause tuboovarian abscess in the literature [4-7].
Diverticulosis is a common condition in elderly population. It is defined as a pocket of mucosa which herniates through areas of weakness usually at vascular entry sites [8]. One of the complications of diverticulosis is diverticulitis which will develop in approximately $10 \%$ of the patients [9]. Occasionally acute diverticulitis will involve the female reproductive organs which lie in close proximity to the sigmoid colon resulting in tuboovarian abscess.

A case of tuboovarian abscess associated with diverticulitis and treated with percutaneous drainage in addition to antibiotic therapy in a young virgin patient with morbid obesity was reported.

\section{Case Presentation}

A 30-year-old virgin patient presented with pelvic pain, fever, and vaginal spotting for the previous three weeks. She was also suffering from malodorous vaginal discharge and 




(a)

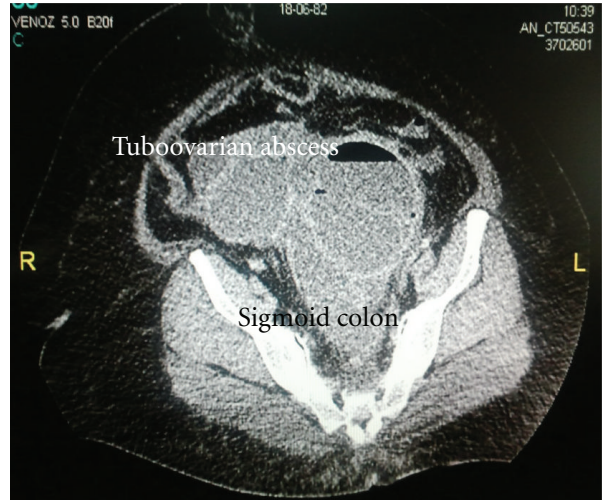

(b)

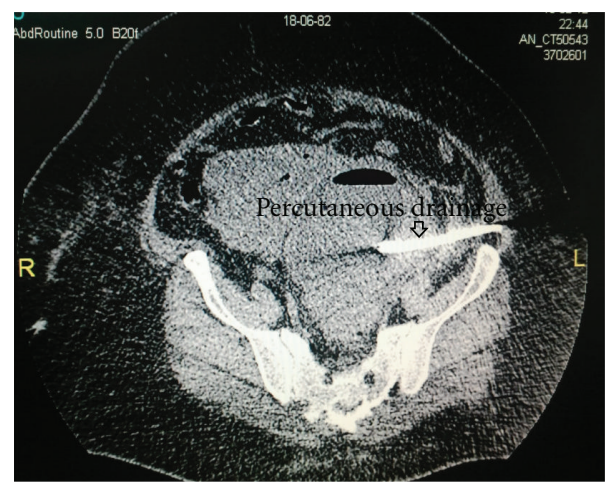

(c)

Figure 1: (a) The sigmoid colon was lying between two tuboovarian masses, and its borders could not be distinguished from the ovaries. (b) The sigmoid colon was lying between two tuboovarian masses. (c) Percutaneous drainage.

constipation. Her past medical history was unremarkable except for morbid obesity. The patient was $160 \mathrm{~kg}$ with a BMI of 35.5 .

Physical examination demonstrated fever $\left(39^{\circ} \mathrm{C}\right)$ and mild tachycardia (110/minute) with bilateral lower abdominal quadrant tenderness. Her blood analysis revealed an elevated white blood count $\left(20.600 / \mathrm{mm}^{3}\right)$ and anemia $(8.5 \mathrm{~g} / \mathrm{dL})$. Her erythrocyte sedimentation rate was $90 \mathrm{~mm} / \mathrm{h}$. Pelvic ultrasonography showed bilateral cystic pelvic mass, but the result was considered to be unsatisfactory due to morbid obesity. CA 125 level was $233 \mathrm{IU} / \mathrm{mL}$. Her blood and urine cultures were found to be negative.

Her abdominopelvic computed tomography scan revealed bilateral complex adnexal masses. The left and right lesions were measured to be $19 \times 11 \mathrm{~cm}$ and $8.5 \times 10 \mathrm{~cm}$ in size, respectively. Both of the lesions were multiseptated with prominent air-fluid levels suggesting tubo-ovarian abscesses (Figures 1(a), 1(b), and 1(c)). The sigmoid colon was lying between two tuboovarian masses, and its borders could not be distinguished from the ovaries. The mesentery of the sigmoid colon was also found to be thickened due to inflammation. The uterus was found to be normal without any sign of pelvic inflammatory disease.

The patient was presumed to have bilateral tuboovarian abscesses which developed as a complication of the sigmoid diverticulitis. Intravenous fluid replacement was started, and oral intake was avoided. Since the patient had fever, tachycardia, and elevated white blood count, she was administered piperacillin-tazobactam by the department of infection diseases considering the risk of sepsis with a dose of $4.5 \mathrm{~g}$ four times a day intravenously. Percutaneous drainage under ultrasonographic guidance was performed using two drainage catheters. A total of $1000 \mathrm{~mL}$ purulent material was aspirated from both lesions. The catheters were left in place for further drainage. Three different bacteria including Escherichia coli, Veillonella, and Peptostreptococcus grew on the cultures of the abscess drainage fluid. During the followup, the clinical condition of the patient gradually improved. She became afebrile, and her white blood count returned to normal level. She was allowed oral intake on the 7th day of hospitalization. Serial ultrasonograms were performed, and decrease in abscess sizes was noted. On the 19th day of hospitalization, the drainage catheters were removed. The patient was discharged on 22 nd day of hospitalization with prominent clinical and radiological improvement.

\section{Discussion}

Since tuboovarian abscess is almost always a complication of pelvic inflammatory disease, it is rarely observed in virgins. However, a review of the literature revealed that 
tuboovarian abscess may also develop due to other rare causes including poor perineal hygiene, previous gynecologic surgery, coexisting genital malignancy, appendicitis, and diverticulitis [4-7].

The possibility of diverticulitis as an etiology of tuboovarian abscess is not usually considered initially. Diverticulitis is usually observed in elderly population, but it may also be diagnosed in younger patients with less incidences. According to [10], the $55-66 \%$ of the women with diverticular disease were over 80 years of age and only $10 \%$ were younger than 40 years of age. Recently, the frequency of diverticular colonic disease increased in patients younger than 50 years of age.

A 31-year-old woman was reported to undergo left salpingo-oophrectomy for a tuboovarian abscess, but she underwent a second surgery because of a recurrent pelvic abscess due to underlying diverticulitis which was undiagnosed at the first operation [11]. Diverticulitis with abscess formation can be very insidious, and reports of presentation as only chronic diarrhea and even brain abscess have been described [12]. A recent case of 63-year-old patient with diverticulitis misdiagnosed to have a primary gynecological abscess subsequently developed colonic perforation [13].

Pelvic pain, signs of infection, and mass at ultrasonography in the presented case easily made the diagnosis of bilateral tuboovarian abscess. Since the patient is virgin, rare causes of tuboovarian abscess were investigated. Computed tomography scan revealed the diagnosis of diverticulitis complicated by tuboovarian abscess [14]. Conservative management with antibiotic therapy and percutaneous drainage resulted in significant clinical improvement of the patient. Since the pelvic inflammatory infection is usually caused by the sexually transmitted microorganism $N$. gonorrhoeae and C. trachomatis, isolated microorganisms further confirmed the diagnosis of abscess resulting from diverticulitis.

\section{Conclusions}

Diverticulitis may be a reason for development of tuboovarian abscess in a virgin patient. Early recognition of the condition with percutaneous drainage in addition to antibiotic therapy helps to have an uncomplicated recovery.

\section{References}

[1] Y. Chan, W. Parchment, J. H. Skurnick, L. Goldsmith, and J. J. Apuzzio, "Epidemiology and clinical outcome of patients hospitalized with pelvic inflammatory disease complicated by tubo-ovarian abscess," Infectious Diseases in Obstetrics and Gynecology, vol. 3, no. 4, pp. 135-139, 1995.

[2] J. DeWitt, A. Reining, J. E. Allsworth, and J. F. Peipert, "Tuboovarian abscesses: is size associated with duration of hospitalization and complications?" Obstetrics and Gynecology International, vol. 2010, Article ID Article ID 847041, 5 pages, 2010.

[3] A. E. Washington, S. O. Aral, P. Wolner-Hanssen, D. A. Grimes, and K. K. Holmes, "Assessing risk for pelvic inflammatory disease and its sequelae," Journal of the American Medical Association, vol. 266, no. 18, pp. 2581-2586, 1991.
[4] A. G. Protopapas, E. S. Diakomanolis, S. D. Milingos et al., "Tubo-ovarian abscesses in postmenopausal women: gynecological malignancy until proven otherwise?" European Journal of Obstetrics Gynecology and Reproductive Biology, vol. 114, no. 2, pp. 203-209, 2004.

[5] A. M. Canas, B. Holloran-Schwartz, and T. Myles, "Tuboovarian abscess 12 years after total abdominal hysterectomy," Obstetrics and Gynecology, vol. 104, no. 5, pp. 1039-1041, 2004.

[6] S. D. Marlow and A. R. Lupetin, "Tuboovarian abscess following transvaginal oocyte retrieval for in vitro fertilization: imaging appearance," Clinical Imaging, vol. 19, no. 3, pp. 180181, 1995.

[7] R. C. Vyas, C. Sides, D. J. Klein, S. Y. Reddy, and M. C. Santos, "The ectopic appendicolith from perforated appendicitis as a cause of tubo-ovarian abscess," Pediatric Radiology, vol. 38, no. 9, pp. 1006-1008, 2008.

[8] A. B. West and M. Losada, "The pathology of diverticulosis coli," Journal of Clinical Gastroenterology, vol. 38, no. 5, supplement 1, pp. S11-S16, 2004.

[9] S. R. Freeman and P. R. McNally, "Diverticulitis," Medical Clinics of North America, vol. 77, no. 5, pp. 1149-1167, 1993.

[10] U. R. Wong, J. R. S. Aguirre, J. M. Cruz Reyes, and J. G. Alvarez, "Complicated diverticular colon disease in patients younger than 35 years: report of two cases and literature review.," Cirugia y Cirujanos, vol. 78, no. 2, pp. 167-171, 2010.

[11] J. A. Naliboff and S. J. Longmire-Cook, "Diverticulitis mimicking a tuboovarian abscess: report of a case in a young woman," Journal of Reproductive Medicine for the Obstetrician and Gynecologist, vol. 41, no. 12, pp. 921-923, 1996.

[12] L. Pompeo, D. S. Heller, M. R. Hameed, J. Sama, and B. M. Cracchiolo, "Unilateral chronic tuboovarian abscess secondary to ruptured colonic diverticulum presenting as a brain abscess: a case report," Journal of Reproductive Medicine for the Obstetrician and Gynecologist, vol. 45, no. 2, pp. 145$148,2000$.

[13] Y. Metz and J. Nagler, "Diverticulitis presenting as a tuboovarian abscess with subsequent colon perforation," World Journal of Gastrointestinal Surgery, vol. 3, no. 5, pp. 70-72, 2011.

[14] N. Hiller, T. Sella, A. Lev-Sagi, S. Fields, and S. Lieberman, "Computed tomographic features of tuboovarian abscess," Journal of Reproductive Medicine for the Obstetrician and Gynecologist, vol. 50, no. 3, pp. 203-208, 2005. 


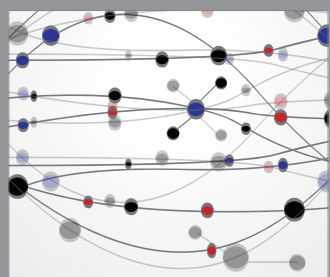

The Scientific World Journal
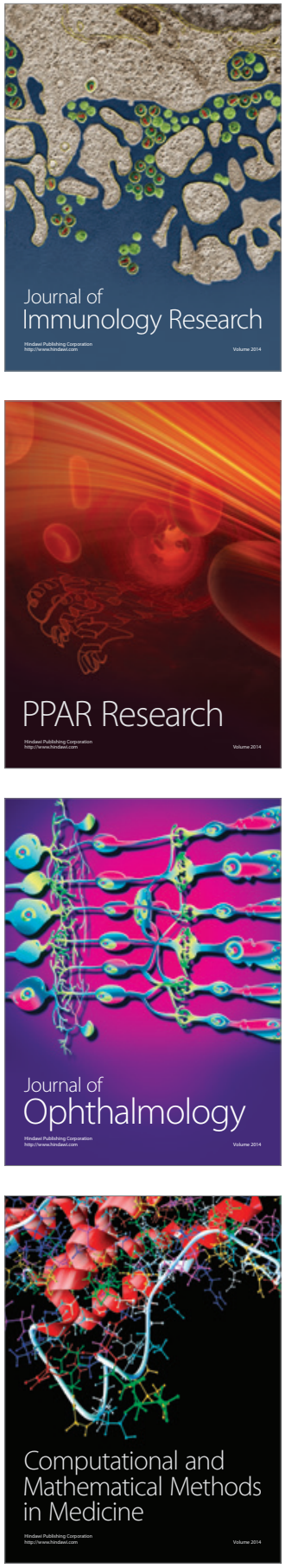

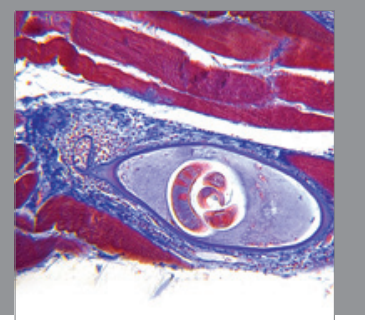

Gastroenterology

Research and Practice
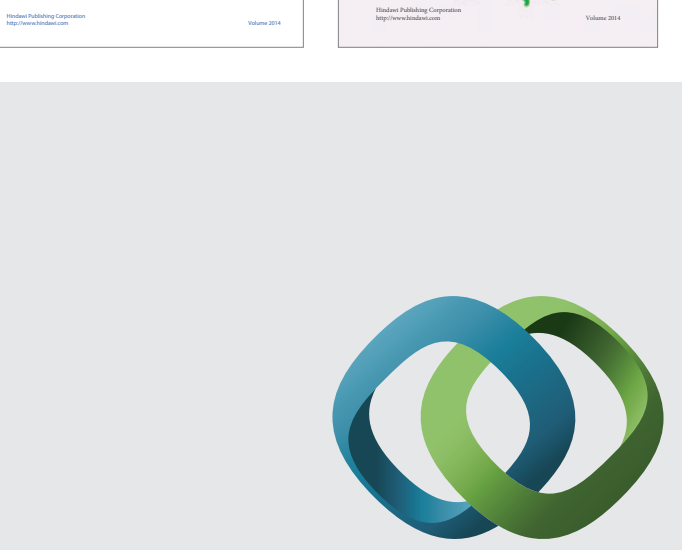

\section{Hindawi}

Submit your manuscripts at

http://www.hindawi.com
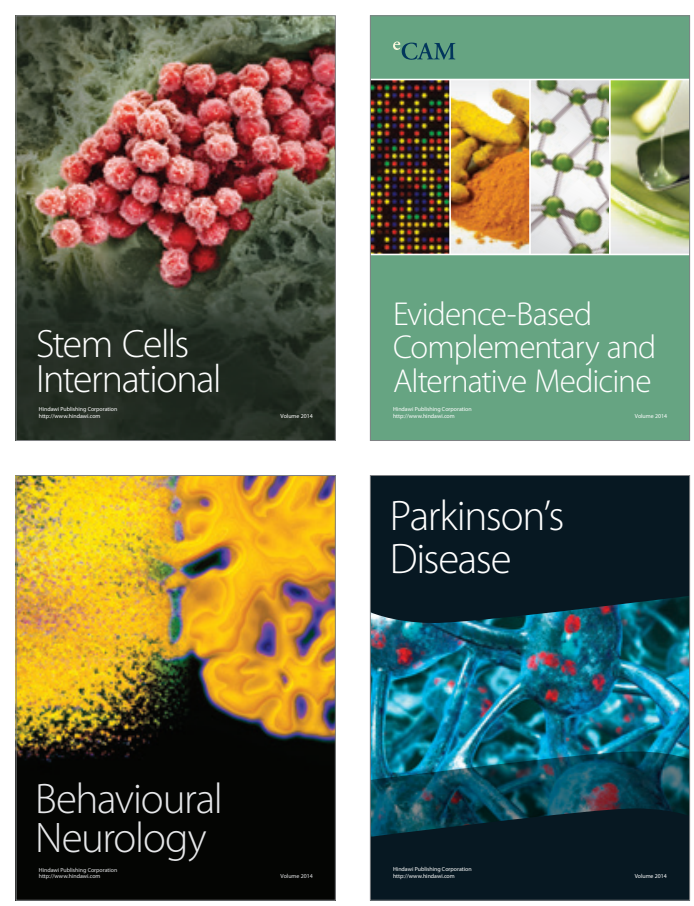

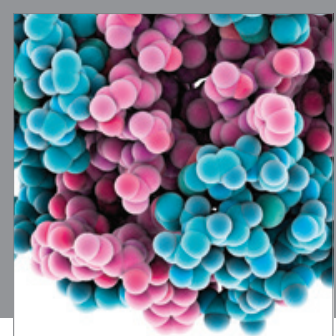

Journal of
Diabetes Research

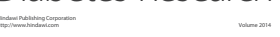

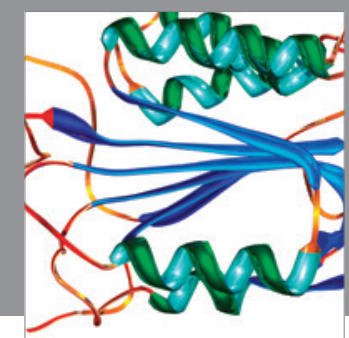

Disease Markers
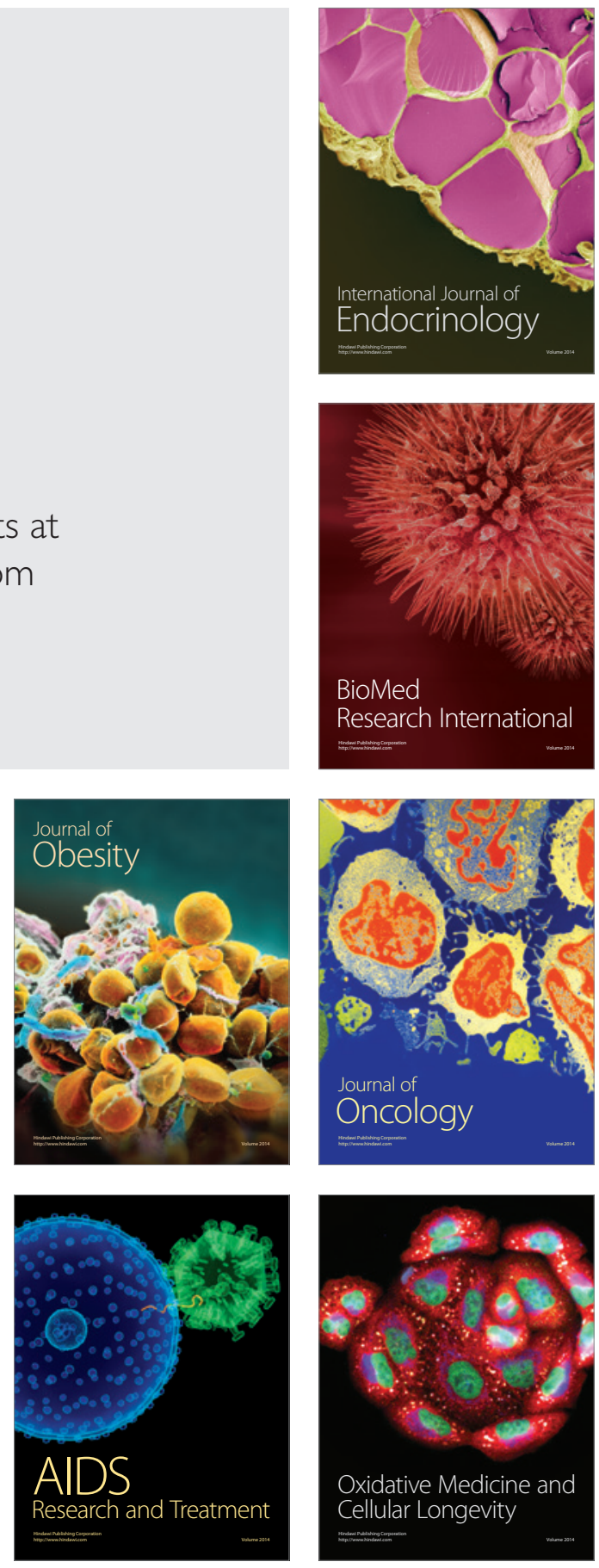